\title{
The Effect of Detection and Restoration Times for Error Recovery in Communication Networks
}

\author{
Dimitris Logothetis* \\ GTE Laboratories Inc. \\ 40 Sylvan Rd. MS 40-A \\ Waltham MA 02254 \\ d100@gte.com
}

\author{
Kishor Trivedi \\ Center for Advanced Comp and Comm. \\ Dept. of Electrical Eng. \\ Duke University Box 90291 \\ Durham NC 27708-0291 \\ kst@ee.duke.edu
}

\begin{abstract}
Detection and restoration times are often ignored when modeling network reliability. In this paper, we develop Markov Regenerative Reward Models (MRRM) to capture the effects of detection and restoration phases of network recovery. States of the MRRM represent conditions of network resources while state transitions represent occurrences of failure, repair, detection and restoration. Reward rates, assigned to states of the MRRM are computed based on a performance model that accounts for contention. We compare our model with ones that ignore these parameters and show significant differences, in particular for transient measures.
\end{abstract}

\section{Introduction}

Communication networks, like any other physical systems, are subject to failures. For this reason, networks are built with special reliability provisions to handle faults. One form of provision is that of a standby resource; when a failure occurs in the active resource, the system is switched to the standby resource while the failed one is being repaired. From the time that a failure occurs until it is actually detected the system performs erroneously. In addition, the switching operation takes a finite amount of time during which system performance is degraded. In high-speed networks, the amount of information per unit time going through a transmission medium is very large. A line failure will imply a large amount of information loss. A major concern in a high speed networking environment, is to keep detection and restoration times very small to minimize information loss.

In this paper, we develop a composite performance and reliability model in order to predict the number of lost messages. The failure-repair model is chosen to be a Markov regenerative one that captures nonexponential detection and reconfiguration time distributions. We note here that the Markov regenerative process is a significant and useful generalization of the semi-Markov process [4]. Some authors [1] have

${ }^{*}$ This work was done while this author was with Duke University. used the term semi-regenerative process to refer to the Markov regenerative process.

Since failure-repair events are rare (when compared to frame interarrival and transmission times) we assume that the system reaches quasi steady-state between successive occurrences of failure-repair events. This observation allows us to separately develop and analyze a contention-based performance model for each state of the failure-repair model. Steady-state performance measures thus computed provide us with reward rates. The stochastic reward model thus captures both the performance and failure effects.

The remainder of the paper is organized as follows: In Section 2 we review the Markov regenerative theory while in Section 3 we describe the protection switching mechanism. In Sections 4 and 5 we develop our dependability models. In Section 6, we present a numerical example and finally in Section 7 we present our conclusions.

\section{Markov Regenerative Reward Mod- els}

Our models are based on the theory of Markov regenerative processes (MRGPs) [1, 4]. Loosely speaking, in a Markov regenerative process there exist time epochs where the process regenerates in the Markovian sense, i.e., the stochastic evolution of the marking process does not depend on the history of the process but rather on the current state. We will consider discretestate continuous time process $\{Z(t), t>0\}$ with state space $\Omega$. Let $v_{i j}(t)$ be the state probabilities conditional on the state of the system at $t=0$, i.e.,

$$
v_{i j}(t)=P\{Z(t)=j \mid Z(0)=i\}, t \geq 0
$$

It can be shown [4] that the conditional probabilities $v_{i j}(t)$ satisfy the following system of integral equations:

$$
v_{i j}(t)=e_{i j}(t)+\sum_{m \in \Omega} \int_{0}^{t} d k_{i m}(u) v_{m j}(t-u)
$$

where $e_{i j}(t), k_{i j}(t)$ are the elements of matrices $\mathbf{K}(t)$ and $\mathbf{E}(t)$ and represent the behavior of the processes 
between regenerations and after a regeneration has occurred, respectively. Note also that matrix $\mathbf{K}(\infty)$ is the transition probability matrix of the embedded DTMC at regeneration points. From matrix V $(t)$ with entries the conditional probabilities $v_{i j}(t)$ and an initial probability vector $\mathbf{p}(0)$ we obtain the unconditional state probabilities as $\mathbf{p}(t)=\mathbf{p}(0) \mathbf{V}(t)$.

The system of integral equations (2) can be converted to a linear system by taking the LaplaceStieltjes transform ${ }^{1}$ on both sides of the equations. We subsequently (numerically) invert the solution of the linear system to obtain state probabilities in timedomain. Due to space limitations we omit the details here. The interested reader could see [6].

In steady-state things are simpler; let $\nu$ be the steady-state probability vector of the embedded DTMC at regeneration points. $\nu$ is the solution of the linear system $\nu=\nu \mathbf{K}(\infty), \sum_{i \in \Omega} \nu_{i}=1$. Let $a_{i j}$ denote the integral $\int_{0}^{\infty} e_{i j}(x) d x$. Then it can be shown under some conditions [4] that the steady-state probabilities $\pi_{j}$ of the MRGP are given by:

$$
\pi_{j}=\frac{\sum_{k \in \Omega} \nu_{k} a_{k j}}{\sum_{k \in \Omega} \nu_{k} \sum_{l \in \Omega} a_{k l}}
$$

Given the system state probabilities at time $t, p_{j}(t)$, $j \in \Omega$ we assign a reward rate $r_{j}$. A variety of measures can be defined based on reward models. In this paper we consider the information loss rate as the reward rate and we will evaluate time-dependent and steady-state expected information loss. In particular, the expected information loss rate at time $t$ is given by:

$$
E[X(t)]=\sum_{j \in \Omega} r_{j} p_{j}(t),
$$

Similarly, we could define the expected accumulated information loss up to time $t$ and the time averaged information loss rate up to time $t[6]$.

\section{$3 \quad$ 1:N protection switching}

Figure 1 shows an $1: \mathrm{N}$ protection switching system [2]. In a $1: \mathrm{N}$ protection switching system, $N$ transmission lines share one common protection line. Each line may be equipped with a buffer to store incoming information (frames). For our performance models, we will consider a finite capacity single server queue, i.e, a queue with $L$ waiting places and one server. Under normal operation, all transmission lines are functioning correctly and the pair of switches connected to line $i$ in cites $\mathrm{A}$ and $\mathrm{C}$ are in the $i$ th position. We define as fault detection time to be the time it takes for the transmitting end to detect the failure. Fault detection might involve the collection of network parameter statistics, e.g., number of frames received in error in a given interval, notification from the layer below or notification from an adjacent node. When a failure is detected in transmission line $i$ its corresponding switches will go to position 0 . Thus standby line is

\footnotetext{
${ }^{1}$ The Laplace-Stieltjes transform for a function $F(t)$ is defined as $F(s)=\int_{0}^{\infty} e^{-s x} d F(x)$
}

used to transmit messages originally handled by line $i$. Before the standby line is called into service the fault in line $i$ must be detected; a process that can take a non-negligible amount of time. Similarly, detection will be followed by a switching time. A second line failure of any lines $0,1, \cdots, i-1, i+1, \cdots, N$ while line $i$ is being repaired will imply a system failure. However, since the repair time will be short compared with the time to failure, we expect system failure to occur very infrequently. Often, when developing de-

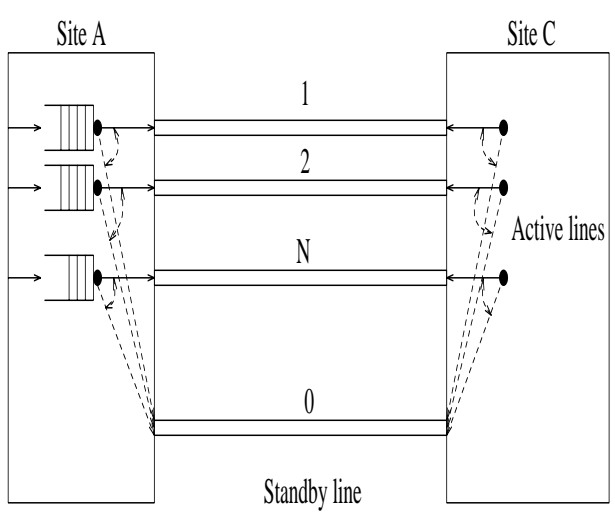

Figure 1: 1:N protection switching

pendability models, detection and switching times are ignored. The purpose of this paper is to develop more accurate models that capture the effects of these times and compare these models with the ones that ignore these times.

\section{Model with instantaneous detection and restoration}

Figure 2 shows the Continuous Time Markov Chain (CTMC) corresponding to system of Figure 1 ignoring detection and restoration times and assuming that times-to-failure and repair times are exponentially distributed random variables. We will also assume one repair person. State $S_{2}$, represents the state where all $N$ transmission lines are operational, while in state $S_{1}$ the standby line has replaced a failed active line. Finally, state $S_{0}$, corresponds to a system configuration with two line failures. Neglecting (temporarily) losses

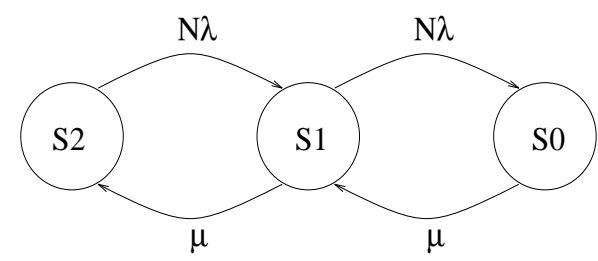

Figure 2: CTMC ignoring detection and restoration times

due to buffer overflow, we associate loss with state 
0 only. Using the time-dependent solution of this $3-$ state CTMC we obtain the time dependent expected loss rate:

$$
\begin{aligned}
r_{\text {loss }}(t) & =\left[\frac{\left(\frac{N \lambda}{\mu}\right)^{2}}{1+\frac{N \lambda}{\mu}+\left(\frac{N \lambda}{\mu}\right)^{2}}-K_{1} e^{-\rho_{1} t}-K_{2} e^{-\rho_{2} t}\right] \times B \\
& =p_{0}(t) \times B
\end{aligned}
$$

where $K_{1}=\frac{\left(\mu-\rho_{1}\right)\left(2 N \lambda+\mu-\rho_{2}\right)-N \lambda \mu}{\left(\rho_{1}-\rho_{2}\right) \rho_{1}}, \quad K_{2}=$ $\frac{\left(\mu-\rho_{2}\right)\left(2 N \lambda+\mu-\rho_{1}\right)-N \lambda \mu}{\left(\rho_{2}-\rho_{1}\right) \rho_{2}}$ and $\rho_{1}, \rho_{2}$ are given by: $(N \lambda+$ $\mu) \pm \sqrt{N \lambda \mu}$. As $t$ tends to infinity $r_{l o s s}(t)$ becomes:

$$
\lim _{t \rightarrow \infty} r_{l o s s}(t)=\left[\frac{\left(\frac{N \lambda}{\mu}\right)^{2}}{1+\frac{N \lambda}{\mu}+\left(\frac{N \lambda}{\mu}\right)^{2}}\right] \times B
$$

In the above equations, $B$ represents the capacity (transmission rate) of the line.

\section{Models with non-zero detection and restoration times}

Figure 3, shows the Markov regenerative reward model [4] in the case with non-zero detection and restoration times. Since failure rates are usually much smaller than repair rates we assume that a second failure cannot occur during the first one's detection and restoration phase. However, we allow for repairs to occur during the detection time of the second failure. States $S_{2}, S_{1}$ and $S_{0}$ have the same meanings as in

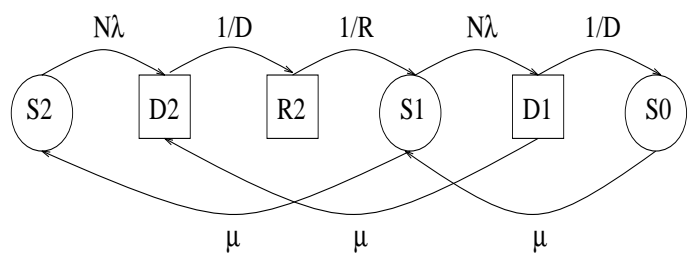

Figure 3: MRGP including detection and restoration times

Figure 2. Sojourn times in states $D_{2}, R_{2}$ and $D_{1}$ are generally distributed with means $D, R$ and $D$ respectively. These additional states, $D_{2}, R_{2}$ and $D_{1}$ can be explained as follows: When a failure occurs, the system takes a finite amount of time to detect it. This delay is captured by the sojourn time in state $D_{2}$. When the failure is detected, the restoration mechanism will be put in effect. The sojourn time in state $R_{2}$, represents the duration of the restoration phase. If a second failure occurs and the first one has not yet been repaired, after the detection phase $D_{1}{ }^{2}$ the system fails, (transition into state 0 ) since no other standby component is available for failure recovery. It can be shown that the stochastic process $\{Z(t), t>0\}$

\footnotetext{
${ }^{2}$ Detection phase in state 1 , can be followed by an unsuccessful restoration phase $R_{1}$.
}

defined as system state at time $t$ is a Markov regenerative process

Let $\left(S_{2}, D_{2}, R_{2}, S_{1}, D_{1}, S_{0}\right)$ label as $(0,1,2,3,4,5)$, for convenience. Let $F_{D}(\cdot)$ and $F_{R}(\cdot)$ denote the distributions of the detection and restoration times respectively.

Due to space limitations we do not include here the structure of matrices $\mathbf{K}(t)$ and $\mathbf{E}(t)$. Once again the interested reader is encouraged to see [6].

We will now discuss the state reward assignment. We assume that information flows into the working lines at a rate of $B$ information units per second. We consider two cases;

No queueing

When no queueing is present, reward assignment (loss rate in this case) would be: $r_{D_{2}}=r_{R_{2}}=r_{D_{1}}=r_{S_{0}}=$ $B$. Reward rates for all remaining states are set to zero. Then, loss rate is given by the expected steadystate reward rate as:

$$
r_{l n b}(t)=B\left[\pi_{D_{2}}(t)+\pi_{R_{2}}(t)+\pi_{D_{1}}(t)+\pi_{S_{0}}(t)\right]
$$

\section{Queueing is present}

We now define two types of losses in the information transmission; losses due to buffer overflow and losses due to channel failures. Buffer overflows can occur in system up states. Let $r_{v}$ denote the probability that an arriving frame will find the buffer full. In both states $S_{2}$ and $S_{1}$ reward rate is defined as $r_{v} \times B$. During the detection phase, the network assumes normal operation. All information is sent to the channel. An incoming frame will be lost either because the buffer was full, (probability $r_{v}$ ), or because its waiting time in the queue was smaller than the detection time:

$$
r_{D_{2}}=B\left[r_{v}+\left(1-r_{v}\right) \int_{0}^{\infty} F_{W}(x) d F_{D}(x)\right]
$$

where $F_{W}(x)$ and $F_{D}(x)$ are the distributions of waiting (in the queue) and detection times respectively.

In communication systems information is transmitted in fixed-size packets. Of interest is the waiting time distribution of the $\mathrm{M} / \mathrm{D} / 1 / \mathrm{L}+1$ queue. In [6] using the results of $[5]$ for the finite capacity $\mathrm{M} / \mathrm{G} / 1$ queue we obtain a closed-form solution for the waiting time distribution in an $\mathrm{M} / \mathrm{D} / 1 / \mathrm{L}+1$ queue as:

$F_{W}(t)= \begin{cases}\frac{\sum_{i=0}^{L}(-1)^{i} \frac{[\lambda(t-i \tau)]^{i}}{i !} e^{\lambda(t-i \tau)} u(t-i \tau)}{\sum_{i=0}^{L}(-1)^{i} \frac{[(i \rho)]^{i}}{i !} e^{(i \rho)}} & 0 \leq t \leq L \tau \\ 1 & t>L \tau\end{cases}$

where:

$$
u(t)= \begin{cases}1 & t \geq 0 \\ 0 & \text { otherwise }\end{cases}
$$

and $\rho=\lambda \tau$.

During restoration, the network has detected the failure and it disables all transmissions. If there are $i$ frames already in the system, more than $L-i+1$ 
arrivals during the restoration phase will contribute to loss. Hence,

$$
r_{R_{2}}=B\left(\sum_{i=0}^{L+1}\left[\sum_{j=L-i+2}^{\infty} \int_{r} A(j, r) d F_{R}(r)\right] p_{i}\right)
$$

where, $A(j, r)$ is the probability of having $j$ arrivals in $r$ time units, $F_{R}(r)$ is the distribution of the restoration time and $p_{i},(i=0,1, \cdots, L+1)$ is the (steadystate) queue length distribution at arrival epochs. Finally, for states $D_{1}$ and $S_{0}$ we associate a reward rate equal to $B$. From the above analysis we obtain:

$$
\begin{aligned}
r_{l o s s}(t) & =B r_{v}\left[\pi_{S_{2}}(t)+\pi_{S_{1}}(t)\right]+r_{D_{2}} \pi_{D_{2}}(t) \\
& +r_{R_{2}} \pi_{R_{2}}(t)+B \pi_{S_{0}}(t)+B \pi_{D_{1}}(t)
\end{aligned}
$$

We can also modify the model of Figure 3 to allow for imperfect coverage of the restoration procedure. This is shown in Figure 4 where $c$ denotes the probability that the restoration procedure is successful (coverage factor).

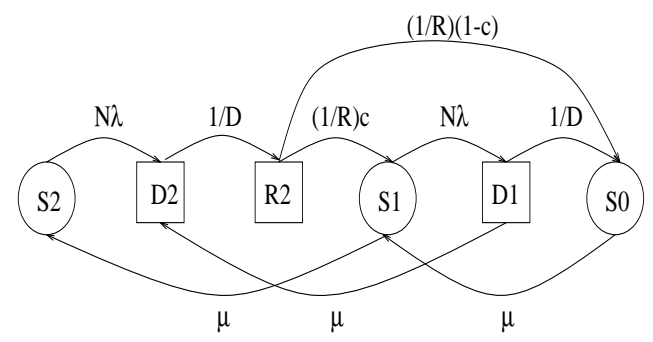

Figure 4: MRGP to allow for imperfect coverage

\section{An Example}

We will consider an example with deterministically distributed detection and restoration times; i.e., $F_{D}(x)=u(t-d)$ and $F_{R}(x)=u(t-r)$. For the steady-state analysis, we compute $\pi_{j}$ using Equation (3). Having obtained the failure-repair model state probabilities we now compute the steady-state loss rate considering the existence of a queue.

\section{No queueing}

Using Equation (7) we obtain the steady-state loss rate for deterministic detection and restoration as:

$$
\begin{aligned}
r_{l n b} & =B\left[\frac{d(\mu+N \lambda)+r\left[N \lambda\left(1-e^{-\mu d}\right)+\mu\right]}{\Delta}\right. \\
& \left.+\frac{\frac{N \lambda}{\mu}\left[1-c\left(1-e^{-\mu d}\right)\right]+1-c}{\Delta}\right]
\end{aligned}
$$

where:

$$
\begin{aligned}
\Delta & =\frac{\mu}{N \lambda}+d(\mu+N \lambda)+r\left[N \lambda\left(1-e^{-\mu d}\right)+\mu\right] \\
& +\frac{N \lambda}{\mu}\left[1-c\left(1-e^{-\mu d}\right)\right]+2-c
\end{aligned}
$$

Note that as $c$ tends to 1 and $d, r$ tend to 0 the above expression becomes identical to Equation (6). In Table 1 we show normalized loss rates $(B=1)$ for different values of detection and restoration times. We assume one active component $(N=1)$, perfect coverage $(c=1), \lambda=10^{-6}$ hours $^{-1}$ and $\mu=1$ hour $^{-1}$.

\begin{tabular}{|c||c||c||c|}
\hline \multirow{2}{*}{\multicolumn{1}{c||}{$\begin{array}{c}\text { Rest. } \\
\text { time } \\
\text { (msecs) }\end{array}$}} & $\begin{array}{c}\text { Normalized loss rate } \\
\text { msecs }\end{array}$ & $\begin{array}{c}\mathrm{D}=100 \\
\text { msecs }\end{array}$ & $\begin{array}{c}\mathrm{D}=1 \\
\mathrm{sec}\end{array}$ \\
\cline { 2 - 4 } & $10^{-12}$ & $2.88 \times 10^{-11}$ & $2.78 \times 10^{-10}$ \\
\hline 20 & $6.55 \times 10^{-12}$ & $3.43 \times 10^{-11}$ & $2.84 \times 10^{-10}$ \\
\hline 60 & $1.77 \times 10^{-11}$ & $4.54 \times 10^{-11}$ & $2.95 \times 10^{-10}$ \\
\hline 100 & $2.88 \times 10^{-11}$ & $5.65 \times 10^{-11}$ & $3.06 \times 10^{-10}$ \\
\hline $1 \mathrm{sec}$ & $2.78 \times 10^{-10}$ & $3.06 \times 10^{-10}$ & $5.56 \times 10^{-10}$ \\
\hline
\end{tabular}

Table 1: Loss rates for different values of detection and restoration times

A question that may naturally arise from Table 1 is for what values of detection and restoration times the effect of these times are insignificant with respect to the measures of interest. By comparing Equations (13) and (6) we see that:

$$
\frac{\left|r_{n l b}-r_{l o s s}(\infty)\right|}{r_{l o s}(\infty)}>\epsilon \Rightarrow d+r>\epsilon \frac{N \lambda}{\mu^{2}}
$$

For example, if $N \lambda \mu=10^{-6}$ a relative difference of at least 9 can be achieved with $d=r=20$ msecs.

In Table 2, we show normalized loss rates for different values of coverage factor and $N$. Detection times are taken to be $D=50$ msecs and the restoration is taken to be 20 msecs.

\begin{tabular}{|c||c||c||c|}
\hline \multicolumn{1}{|c||}{$\begin{array}{c}\text { Cov } \\
\text { factor }\end{array}$} & \multicolumn{3}{c|}{ Normalized loss rate } \\
\cline { 2 - 4 } & $\mathrm{N}=1$ & $\mathrm{~N}=4$ & $\mathrm{~N}=16$ \\
\hline 0.85 & $1.5 \times 10^{-7}$ & $6.00 \times 10^{-7}$ & $2.4 \times 10^{-6}$ \\
\hline 0.90 & $1.0 \times 10^{-7}$ & $4.0 \times 10^{-7}$ & $1.6 \times 10^{-6}$ \\
\hline 0.95 & $5.0 \times 10^{-8}$ & $2.0 \times 10^{-7}$ & $8.0 \times 10^{-7}$ \\
\hline 1.0 & $2.04 \times 10^{-11}$ & $9.38 \times 10^{-11}$ & $5.67 \times 10^{-10}$ \\
\hline
\end{tabular}

Table 2: Loss rates for different values of detection and restoration times

For the transient measures of Figure 5 we used numerical transform inversion based on Jagerman's method [3]. Figure 5 illustrates a comparison of the Markovian model with instantaneous detection / restoration and the one with non-zero detection and restoration times. Note the significant differences in particular for transient measures.

Queueing is present

We assume Poisson arrivals with rate $\alpha$ and deterministically distributed service times. Using Equation 


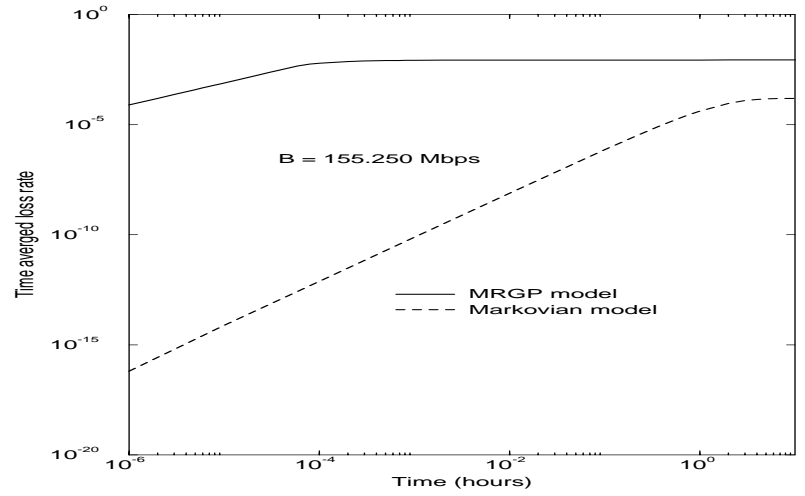

Figure 5: Comparison of the Markovian and the nonMarkovian model

(9) we compute the response time distribution of the $\mathrm{M} / \mathrm{D} / 1 / \mathrm{L}+1$ queue that is needed for the evaluation of $r_{D_{2}}$ according to Equation (8). We will also assume that detection and restoration times are deterministic. For $\rho=\alpha \tau=0.5, L+1=20, \tau=1 \mathrm{msec}, d=r=10$ msecs we get: $r_{D_{2}}=0.999998 \times B, r_{R_{2}}=1.79 \times 10^{-6} B$ and finally $r_{\text {loss }}=2.07 \times 10^{-11} B$. Figure 6 shows the normalized loss rate $(B=1)$ for three different values of detection and restoration phases. Finally, Figure 7

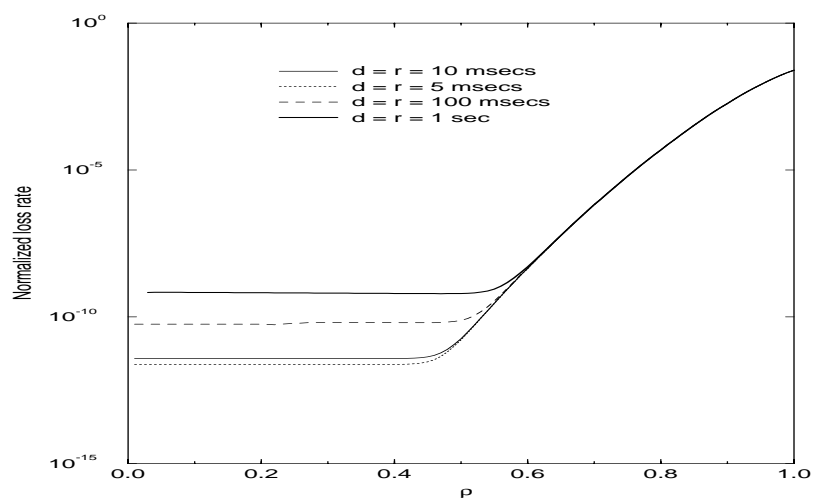

Figure 6: Steady-state normalized loss rate as a function of load $\rho$

shows the effect of buffer size on the normalized loss rate. Note that for small values of buffer size, the probability of overflow is the dominating factor when quantifying the normalized loss rate but as the buffer size increases, the contribution of buffer overflow becomes very small, and furthermore the normalized loss rate becomes insensitive to the buffer size.

\section{Conclusions}

In this paper, we presented a Markov regenerative reward model to capture the effects of detection and restoration in protection switching networks. This allowed us to capture realistic aspects of system behavior such as non-exponential detection and restoration times. Loss of messages during detection time and reduced performance during reconfiguration time are also accounted for. Incorporation of reward rates in

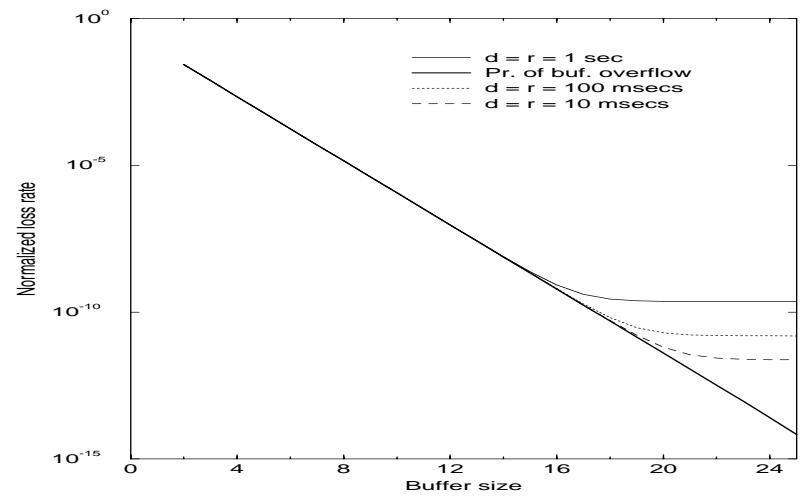

Figure 7: Steady-state normalized loss rate as a function of buffer size

the failure/repair model allows us to incorporate performance aspects and hence calculate system performance in the presence of failures. Performance model chosen is a $\mathrm{M} / \mathrm{D} / 1 / \mathrm{L}+1$ queue. As measures of interest, we considered the time-dependent as well as the steady-state expected loss rate. We also considered cumulative measures such as the expected time-averaged frame loss in an interval of length $T$. The above model can be extended to include a $\mathrm{k}: \mathrm{N}$ protection switching system where $N$ working sections share $k$ (standby) protection sections.

\section{References}

[1] E. Cinlar. Introduction to Stochastic Processes, Prentice Hall, 1975.

[2] CCITT Recommendation G.783. Characteristics of Synchronous Digital Hierarchy (SDH) Multiplexing Equipment Functional Blocks, Annex A, Geneva 1990.

[3] D. Jagerman. An Inversion Technique for the Laplace Transforms. Bell System Technical Journal, Vol. 6, No. 8, 1995-2002, 1982.

[4] V. G. Kulkarni. Lecture Notes on Stochastic Models in Operations Research. University of North Carolina, Chapel Hill, U.S.A., 1990.

[5] S. Lavenberg. The Steady State queueing time distribution for the $\mathrm{M} / \mathrm{G} / 1$ finite capacity queue. Management Science, Vol. 21, January 1975.

[6] D. Logothetis. Transient Analysis of Communication Networks, Ph.D. thesis, Dept. of Electrical Eng., Duke University. 